\title{
Роль психологических и физиологических условий материнства в развитии послеродовой депрессии
}

\section{Вера А. Якупова}

Московский государственный университет имени М.В. Ломоносова, г. Москва, Российская Федерация

E-mail: vera.a.romanova@gmail.com

\begin{abstract}
Аннотация
Введение. В статье рассматриваются психологические и фризиологические условия реализации материнства у женщин, входящих в группу риска развития послероАовой Аепрессии. Приводятся Аанные исслеАОваний факторов риска развития Аепрессии и результаты оригинального исслеАования. ИсслеАОватели в основном СосреАОтачивают внимание на личностных чертах матери, в центре нашей работы стоит изучение отношения женщины к опыту материнства и условий, в которых оно реализуется. Новизна исследования также состоит в разработке и апробации Аиагностического инструментария, направленного на выявление группы риска развития Аепрессии. Методы. В качестве метоАОв исслеАОвания использовались ЭАинбургский опросник послеродовой депрессии (Edinburgh Postnatal Depression Scale (EPDS)), шкала $А е-$ прессии Бека (Beck Depression Inventory), авторская анкета об условиях материнства. Результаты. В исследовании приняли участие матери ( $n=151)$ млаАенцев Ао года. Основываясь на полученных Аанных, удалось вылелить группу риска развития послеродовой Аепрессии. В эту группу входят матери с Аепривацией сна (меньше 7 часов в сутки), которые не ощущают стабильной подАержки мужа. Матери группы риска пережили сложные роды, а их ожидания от материнства оказались нереалистичными. Приводятся Аанные апробации Аиагностического инструментария. ОбсужАение результатов. Аелается вывоА, что при анализе риска развития послеродовой Аепрессии важно учитывать целый комплекс фракторов: ожиАания женщины относительно материнства и отношение к реальным условиям родительства, контекст супружеских отношений и ффизиологические условия, в которых реализуется родительство. ОбсужАаются возможности практического применения полученных результатов.
\end{abstract}

\section{КАючевые слова}

послеродовая депрессия, психология беременности, психология материнства, психология родительства, шкала Аепрессии Бека, шкала послеродовой Аепрессии, профилактика послеродовой Аепрессии, Аиагностика послеродовой Аепрессии, риск послеродовой Аепрессии, Аетско-родительские отношения 


\title{
Основные положения
}

- опросник Бека и Элинбургская шкала послеродовой Аепрессии являются наАежными инструментами Аиагностики послеродовой Аепрессии;

- женщины, чьи ожидания от материнства оказались нереалистичными, имеют более выраженные показатели депрессии;

- стабильная подАержка супруга снижает риск развития послеродовой Аепрессии; - матери с выраженными признаками депрессии значимо чаще имеют тяжелый опыт родов.

\section{Благодарности}

Исследование выполнено в рамках реализации гранта РФФИ, № 18-313-00051.

\section{Для цитирования}

Якупова В.А. Роль психологических и физиологических условий материнства в развитии послеродовой депрессии // Российский психологический журнал. - 2018. - Т. 15, № 1. - C. 8-23. DOI: 10.21702/rpj.2018.1.1

\section{The Impact of Psychological and Physiological Conditions of Motherhood on Postnatal Depression}

\author{
Vera A. Yakupova \\ Lomonosov Moscow State University, Moscow, Russian Federation \\ E-mail: vera.a.romanova@gmail.com
}

\begin{abstract}
Introduction. The paper (a) discusses psychological and physiological conditions of motherhood among women at risk of postnatal depression, (b) overviews studies on depression risk factors, and (c) presents the results of the original study. This research concentrates on mothers' views of their own experience of motherhood and also their personality traits. The present study develops and validates diagnostic tools for identifying women at risk of depression.

Methods. The study employed the Edinburgh Postnatal Depression Scale (EPDS), Beck Depression Inventory, and the author's questionnaire on motherhood conditions.

Results. The study involved mothers $(n=151)$ of babies under one year old. The conducted study helped to reveal a group of women at risk of postnatal depression. Sleep-deprived mothers (sleeping less than 7 hours per day) not feeling a constant support from their
\end{abstract}


husbands fell into this risk group. The risk group mothers underwent a difficult labor; they had unrealistic expectations of motherhood.

Discussion. The following factors determine the risks of postnatal depression: (a) a woman's expectations of motherhood, (b) her views of parenthood realities, (c) the context of marital relations, and (d) psychological conditions of parenthood. The research findings can be readily used in practice.

\section{Keywords}

postnatal depression, psychology of pregnancy, psychology of motherhood, psychology of parenthood, Beck Depression Inventory, postnatal depression scale, prevention of postnatal depression, postpartum depression diagnosis, risk of postnatal depression, child-parent relationship

\section{Highlights}

- The Edinburgh Postnatal Depression Scale and Beck Depression Inventory are reliable tools for postpartum depression diagnosis.

- Women with unrealistic expectations of motherhood have the highest level of depression.

- A spouse's constant support reduces the risk of postnatal depression.

- Severely depressed mothers more often undergo a difficult labor.

\section{Acknowledgments}

This work was supported by the Russian Foundation for Basic Research, project no. 18-313-00051.

\section{For citation}

Yakupova V.A. The Impact of Psychological and Physiological Conditions of Motherhood on Postnatal Depression. Rossiiskii psikhologicheskii zhurnal - Russian Psychological Journal, 2018, V. 15, no. 1, pp. 8-23 (in Russian). DOl: 10.21702/rpj.2018.1.1

Original manuscript received 21.08.2017

\section{Введение}

Послеродовая депрессия (ПРД), согласно МКБ-10, имеет симптоматику клинической депрессии, но проявляется в течение 6 недель после родов. При этом ПРД может длиться вплоть до года [1]. В европейских странах ПРД встречается с частотой 10-15\% [2], в мусульманских странах - 17-27\% [3]. Наряду с антидепрессантами эффективным средством лечения послеродовой депрессии является психотерапия [4].

Зарубежные исследователи в основном сосредотачивают внимание на выявлении различных факторов, повышающих риск возникновения послеродовой 


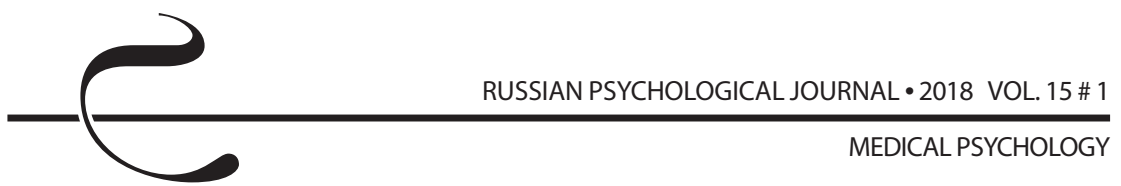

депрессии. К примеру, вероятность возникновения послеродовой депрессии выше у женщин, которые демонстрировали признаки депрессии во время беременности [5]. Есть данные о том, что в развитии депрессии серьезную роль играет тяжелый опыт родов [6]. Отсутствие социальной поддержки [7] и сложное финансовое положение [5] также являются факторами риска развития послеродовой депрессии.

Развитию послеродовой депрессии способствуют такие личностные черты, как перфекционизм, склонность подавлять свои чувства и отсутствие умения просить других о помощи [8]. Нестабильная самооценка также является предиктором развития депрессии после рождения ребенка [9]. Исследования говорят о том, что в развитии депрессии значительную роль играют когнитивные особенности женщины, такие как склонность фиксировать внимание на негативных событиях и руминации [10]. Трудности эмоциональной регуляции также могут лежать в основе депрессии [11]. Немаловажную роль в эмоциональной саморегуляции играет уровень развития регуляторных функций. Есть данные о том, что родители с низким уровнем развития регуляторных функций чаще жалуются на сложности во взаимодействии с ребенком, оказываются более строгими и проявляют меньше терпения в совместной деятельности [12].

Серьезное значение в освоении материнской роли и построении детскородительских отношений играет тип привязанности женщины к собственной матери. Так, риску развития послеродовой депрессии подвержены женщины с ненадежным и тревожно-избегающим типами привязанности [13]. Они чаще испытывают сложности во взаимодействии с ребенком и с супругом, ощущают меньше поддержки со стороны социального окружения.

В наших предыдущих исследованиях было выявлено, что важное значение в процессе освоения роли матери имеют три группы условий: уровень ригидности, оправданность ожиданий относительно материнства и отношение к компонентам этой роли (выполнению родительских функций, к себе в роли матери и эмоциональное отношение к ребенку) [14]. Матери с высоким уровнем ригидности оценивают сложность адаптации к рождению ребенка как наиболее высокую. Сложнее привыкать к изменениям, связанным с рождением ребенка, женщинам, чьи ожидания относительно материнства не оправдались, субъективно ими переживается отказ от многих сфер жизни.

ПРД оказывает влияние не только на благополучие женщины, но и на всю семейную систему. Женщинам, находящимся в состоянии депрессии, сложнее распознавать состояния ребенка и адекватно реагировать на них [11], что, в свою очередь, оказывает негативное влияние на формирование привязанности ребенка к матери, на его эмоциональное и интеллектуальное развитие $[15,16]$. Так, дети матерей, которые перенесли эпизод послеродовой 
депрессии, в возрасте 1,5 лет чаще демонстрируют тревожно-избегающий тип привязанности, чем дети контрольной группы [17]. По данным исследований существует связь между беспокойным темпераментом ребенка - постоянным плачем, плохим сном - и выраженными депрессивными симптомами у матери [18], однако направление этой связи определить сложно - возможно, происходит взаимовлияние реакции матери, которая только усиливает негативные проявления у ребенка.

Послеродовая депрессия оказывает негативное влияние не только на состояние матери и ее отношения с ребенком, но также на процесс освоения родительской роли отцом ребенка. Так, в исследовании L. Beestin et al. [19] говорится о том, что эпизод постнатальной депрессии у матери тяжело переживается отцом ребенка, зачастую приводит к эмоциональному отстранению от ребенка, чувству одиночества и изоляции. Риск развития депрессии у отца напрямую связан с уровнем выраженности депрессии у матери [20].

Таким образом, послеродовая депрессия является опасной не только для матери, но и имеет долгосрочные последствия для психологического благополучия всей семьи. В связи с этим нам представляется важным дальнейшее исследование факторов риска возникновения послеродовой депрессии с целью ее профилактики.

Целью данной работы явилось исследование связи выраженности послеродовой депрессии с физиологическими (сон, кормление, особенности здоровья и т. д.) и психологическими (поддержка близких, ожидания матери и т. д.) условиями материнства, выявление группы риска развития послеродовой депрессии.

\section{Методы}

\section{Выборка}

В исследовании приняли участие матери $(\mathrm{n}=151)$ младенцев в возрасте от 1 недели до года (средний возраст 5,4 месяца). Средний возраст участниц исследования 31,5 лет, среди них 62,9\% - матери первого ребенка, 27,8\% - матери второго ребенка, 9,3\% - многодетные матери. 2 участницы исследования растят ребенка без мужа, 98,6\% участниц замужем.

\section{Методики}

Для определения степени выраженности депрессии в исследовании использовались две методики - шкала депрессии Бека (Beck Depression Inventory) [21] и Эдинбургский опросник послеродовой депрессии (Edinburgh Postnatal Depression Scale (EPDS)) [22]. Русскоязычная версия шкалы Бека опубликована в книге «Эмоции и чувства» Е. П. Ильина [23] со ссылкой на источник - книгу В. Каппони, Т. Новака «Сам себе психолог» [24]. Методика была 


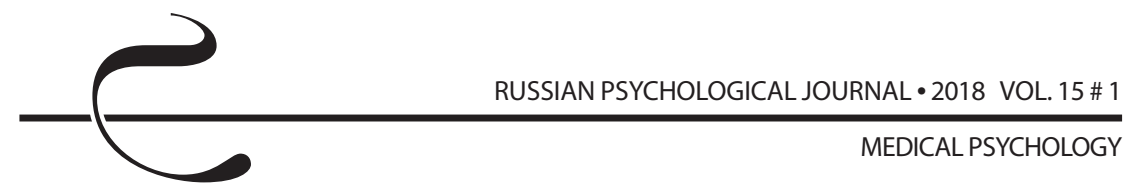

переведена как часть научно-популярной книги. Адаптация на российской выборке данной методики не проводилась. В связи с этим мы осуществили повторный перевод методики совместно с профессиональным переводчиком. Коэффициент альфа Кронбаха показал, что версия методики является надежной $(a=0,866$, при исключении 19-го вопроса $a=0,873)$. Оригинальная методика на английском языке имеет показатель надежности $a=0,86$.

В исследовании Г.Э. Мазо [25] была предпринята попытка адаптации методики EPDS на российской выборке (195 матерей приняли участие в исследовании). Методика показала достаточно высокую надежность $(a=0,73)$. К сожалению, авторами не приводится перевод методики. Мы осуществили перевод оригинальной методики EPDS, который показал высокую надежность $(a=0,838)$.

В нашем исследовании согласованность данных по двум методикам опроснику Бека и EPDS - оказалась высокой ( $r$ Spearman $=0,798, p<0,01$ ).

Также нами была составлена анкета, содержащая следующие параметры:

- возраст матери;

- возраст ребенка;

- количество детей в семье и их возраст;

- информацию о здоровье ребенка. Респонденток просили оценить здоровье ребенка по пятибалльной шкале (1 - нет проблем со здоровьем, 5 - есть серьезные проблемы со здоровьем);

- информацию о способе вскармливания (грудное или искусственное);

- информацию о родах. Респонденток просили оценить сложность родов по пятибалльной шкале (1 - роды прошли легко, 5 - с серьезными осложнениями);

- количество часов сна в сутках;

- субъективное ощущение поддержки со стороны супруга;

- субъективное ощущение поддержки со стороны других родственников;

- долю заботы о ребенке, которая полностью принадлежит матери (в процентах);

- ожидания, связанные с материнством. Респонденток просили оценить, насколько их представления о жизни с ребенком совпали с реальностью, по пятибалльной шкале (1 - полностью совпали, 5 - все оказалось совершенно по-другому).

\section{Результаты}

Описываемые ниже значимые связи были выявлены по данным двух опросников - EPDS и Beck Depression Scale. В описании мы приводим значения связей только c EPDS (поскольку он более специфичен для ПРД) для удобства восприятия. В таблице 1 указаны значения по двум опросникам. 
В нашем исследовании получены данные о связи уровня выраженности послеродовой депрессии с двумя условиями материнства: количеством часов сна матери в сутки (r Spearman =0,296, p <0,01) (таблица 1) и субъективного ощущения поддержки со стороны мужа $(U=6,023, p=0,049)$ (таблица 2). Значимости поддержки других родственников выявлено не было. Также доля заботы о ребенке матери относительно других родственников не связана с депрессией.

Значимым риском развития послеродовой депрессии являются нереалистичные ожидания относительно материнства (r Spearman =0,296, p $<0,01)$. Женщины, чьи ожидания от материнства не совпали с реальностью, имеют более выраженные показатели депрессии. Соответственно, опыт материнства помогает формировать более адекватные реальности ожидания ( $r$ Spearman $=-0,313, p<0,01$ ).

Была обнаружена связь выраженности ПРД с опытом родов: роды с осложнениями сопровождаются более высокими показателями депрессии (r Spearman = 0,216, p <0,01). При этом опыт родов связан с количеством часов сна матери в сутки ( $r$ Spearman $=-0,204, p<0,05)$. Связи выраженности ПРД со здоровьем ребенка не было обнаружено.

Таблица 1. Корреляционные связи условий материнства с показателями Аепрессии по опросникам EPDS и BDS

Table 1. Correlations among conditions of motherhood and depression by the EPDS and BDS

\begin{tabular}{|c|c|c|}
\hline $\begin{array}{l}\text { Условия материнства } \\
\text { Conditions of motherhood }\end{array}$ & EPDS & $\begin{array}{l}\text { Beck Depression } \\
\text { Scale }\end{array}$ \\
\hline $\begin{array}{l}\text { Количество часов сна в сутки } \\
\text { Hours of sleep per day }\end{array}$ & $-0,296^{* *}$ & $-0,307^{* *}$ \\
\hline $\begin{array}{l}\text { Опыт ролов (по пятибалмьной шкале, } \\
\text { гАе } 1 \text { - ^егкие ролы, } 5 \text { - ролы с се- } \\
\text { рьезными осложнениями) } \\
\text { Labor experience (on a five-point } \\
\text { scale, where } 1 \text { means easy labor and } 5 \\
\text { means serious complications of labor) }\end{array}$ & $0,216^{* *}$ & $0,212^{* *}$ \\
\hline $\begin{array}{l}\text { Ожилания от материнства } \\
\text { Expectations of motherhood }\end{array}$ & $0,296^{* *}$ & $0,267^{* *}$ \\
\hline
\end{tabular}

Примечание: критерий r Spearman, $p<0,01$.

Note: Spearman's coefficient of rank correlation, $p<0.01$ 
Таблица 2. Связь условий материнства с показателями депрессии по опросникам EPDS и BDS, по критерию Манна - Уитни

Table 2. The relationship among conditions of motherhood and depression by the EPDS and BDS (Mann-Whitney U test)

\begin{tabular}{|c|c|c|}
\hline $\begin{array}{l}\text { Условия материнства } \\
\text { Conditions of motherhood }\end{array}$ & EPDS & Beck Depression Scale \\
\hline $\begin{array}{l}\text { Подлержка супруга } \\
\text { A spouse's support }\end{array}$ & $U=6,023 ; p=0,049$ & $U=9,511 ; p=0,009$ \\
\hline
\end{tabular}

На основании полученных данных была проведена статистическая процедура кластерного анализа с целью выявить группу риска развития депрессии. В ходе анализа данные о респондентках были разделены на три кластера:

1. Отсутствие депрессии (67 матерей).

2. Пограничное состояние, выраженные признаки депрессии (65 матерей).

3. Тяжелая депрессия (19 матерей).

Между кластерами были обнаружены значимые различия по показателям депрессии и указанным выше параметрам: сложность родов, количество часов сна матери в сутки, ожидания относительно материнства и ощущение поддержки со стороны мужа (таблица 3). В таблице 4 представлены средние значения данных параметров для трех кластеров.

Из приведенных в таблице 3 данных можно увидеть, что кластер 1 (отсутствие депрессии) контрастно отличается от кластера 2 (выраженная депрессия) и кластера 3 (тяжелая депрессия) по всем выделенным параметрам. Матери с выраженной депрессией и тяжелой депрессией значимо различаются только по показателям опросников EPDS и Beck Depression Scale.

По данным, приведенным в таблице 4, удалось выделить группу риска развития послеродовой депрессии. В эту группу входят матери, которые спят меньше 7 часов в сутки, не ощущают стабильной поддержки мужа. Роды у матерей группы риска прошли с осложнениями, а их ожидания от материнства оказались нереалистичными.

Статистически значимых различий не было выявлено, однако на уровне тенденции обнаружилась следующая закономерность: чаще в группу риска развития депрессии также попадают матери, у которых есть старшие дети раннего возраста и младенцы которых находятся на грудном вскармливании. 


\begin{tabular}{|c|c|c|c|c|c|c|}
\hline & 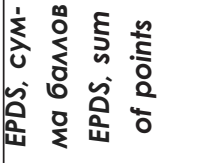 & $\begin{array}{ll}0 & 8 \\
0 & 0 \\
0 & 0 \\
1 & 0 \\
11 & 11 \\
0 & 0\end{array}$ & & $\begin{array}{l}8 \\
\text { ¿ } \\
0 \\
11 \\
2\end{array}$ & & \begin{tabular}{ll}
\multirow{2}{*}{} & 8 \\
0 & 0 \\
0 & 0 \\
11 & 11 \\
11 & 0
\end{tabular} \\
\hline & 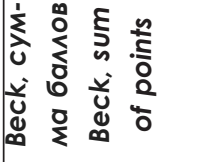 & $\begin{array}{ll}0 & 8 \\
0 & 0 \\
0 & 0 \\
1 & 0 \\
11 & 11 \\
0 & 0\end{array}$ & 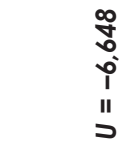 & $\begin{array}{l}8 \\
8 \\
0 \\
11 \\
2\end{array}$ & & \begin{tabular}{ll}
$\infty$ & 8 \\
\multirow{2}{*}{} & 8 \\
0 & 0 \\
1 & 11 \\
11 & 0 \\
$>$ & 0
\end{tabular} \\
\hline$\frac{ᄃ}{\overline{0}}$ & 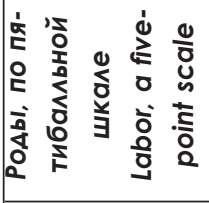 & 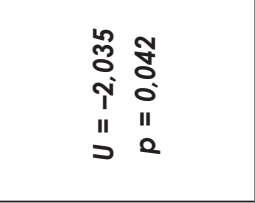 & 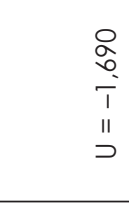 & $\begin{array}{l}\bar{a} \\
\text { Oे } \\
\text { o } \\
11 \\
0\end{array}$ & & 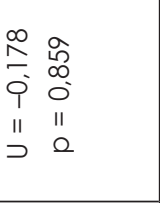 \\
\hline $\begin{array}{l}\frac{0}{0} \\
\frac{0}{0} \\
\frac{0}{0} \\
\frac{0}{1} \\
\frac{1}{0} \\
\frac{0}{2} \\
0 \\
\frac{0}{0} \\
i \\
\frac{1}{E}\end{array}$ & 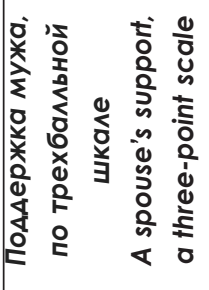 & 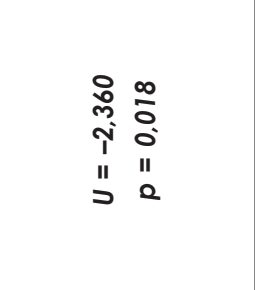 & 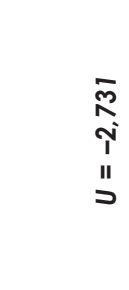 & $\begin{array}{l}\text { ¿ } \\
0 \\
11 \\
2\end{array}$ & & 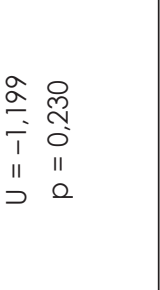 \\
\hline 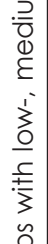 & 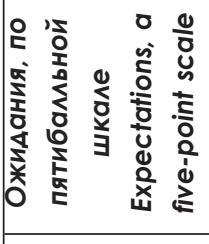 & \begin{tabular}{ll}
$\infty$ & $\overline{8}$ \\
\multirow{4}{*}{} & 0 \\
1 & 0 \\
1 & 11 \\
11 & 0
\end{tabular} & $\begin{array}{l}\overline{1} \\
\text { o } \\
\text { p) } \\
\text { II } \\
0\end{array}$ & $\begin{array}{l}\text { 尺े } \\
\text { o } \\
\text { "1 } \\
2\end{array}$ & & \begin{tabular}{ll}
0 & 0 \\
& 2 \\
\hdashline 1 & 0 \\
11 & 11 \\
1 & 0
\end{tabular} \\
\hline 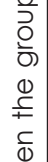 & 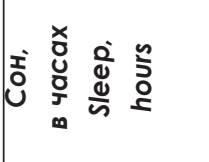 & 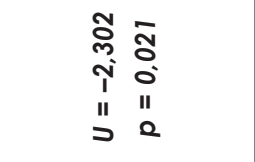 & $\begin{array}{l}\text { ָ̃ } \\
\text { N } \\
\text { II } \\
\nu\end{array}$ & $\begin{array}{l}\hat{8} \\
0 \\
0 \\
11 \\
2\end{array}$ & & \begin{tabular}{ll}
$\stackrel{2}{n}$ & 0 \\
& 0 \\
\hdashline & 0 \\
1 & 0 \\
11 & 11 \\
$\supset$ & 0
\end{tabular} \\
\hline 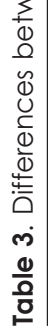 & & 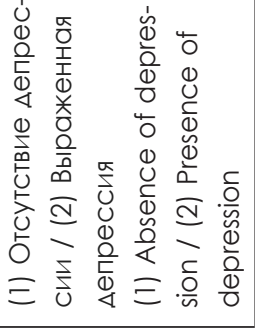 & 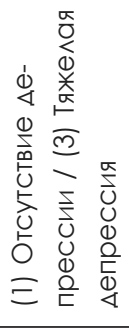 & 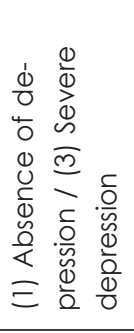 & 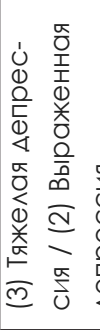 & 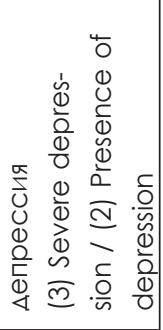 \\
\hline
\end{tabular}


Таблица 4. Характеристика групп с низкой, средней и высокой выраженностью мепрессии

Table 4. Characteristics of the groups with low-, medium-, and high-level depression

\begin{tabular}{|c|c|c|c|c|}
\hline & $\begin{array}{c}\text { Сон, } \\
\text { в часах } \\
\text { Sleep, hours }\end{array}$ & $\begin{array}{c}\text { Ожилания, } \\
\text { по пяти- } \\
\text { бам^ьной } \\
\text { шкале } \\
\text { Expectations, } \\
\text { a five-point } \\
\text { scale }\end{array}$ & $\begin{array}{c}\text { ПодАержка } \\
\text { мужа, по } \\
\text { трехбаммьной } \\
\text { шкале } \\
\text { A spouse's sup- } \\
\text { port, a three- } \\
\text { point scale }\end{array}$ & $\begin{array}{c}\text { Роды, } \\
\text { по пяти- } \\
\text { бамльной } \\
\text { шкале } \\
\text { Labor, a } \\
\text { five-point } \\
\text { scale }\end{array}$ \\
\hline $\begin{array}{l}\text { (1) Отсутствие } \\
\text { Аепрессии } \\
\text { (1) Absence of } \\
\text { depression }\end{array}$ & 7,11 & 2,28 & 1,66 & 1,85 \\
\hline $\begin{array}{l}\text { (2) Выраженная } \\
\text { Аепрессия } \\
\text { (2) Presence of } \\
\text { depression }\end{array}$ & 6,54 & 3,05 & 1,31 & 2,31 \\
\hline $\begin{array}{l}\text { (3) Тяжелая } \\
\text { Аепрессия } \\
\text { (3) Severe } \\
\text { depression }\end{array}$ & 5,91 & 3,47 & 1,05 & 2,32 \\
\hline
\end{tabular}

\section{Обсуждение результатов}

Опросник Бека и Эдинбургская шкала послеродовой депрессии в нашем переводе показали себя как надежные инструменты диагностики ПРД. Необходимо апробировать методики на более широкой выборке.

Проведенное исследование убедительно показывает, что отсутствие реалистичных ожиданий является одним из факторов риска развития послеродовой депрессии. Результаты исследования соотносятся с ранее полученными нами данными о важной роли ожиданий в процессе освоения материнской роли [14]. Идеализация материнства связана со сложностями в адаптации к выполнению родительских функций. В исследовании T. Muscat et. al [26] высказана гипотеза о том, что стратегия «ожидания худшего» является наиболее эффективной - родители, ожидавшие сложностей с кормлением и сном ребенка, вошли в наиболее благополучную группу с самым низким уровнем постнатальной депрессии. По-видимому, стратегия «ожидания худшего» позволяет родителям быть психологически готовыми к трудностям. 
Тяжелые роды могут оказывать влияние на здоровье ребенка и, соответственно, вызывать проблемы со сном - в нашем исследовании обнаружена связь между количеством часов сна матери и субъективной тяжестью родов. Однако негативный опыт родов может быть фактором риска развития депрессии вне связи сложностей со сном ребенка. Так, в исследовании T. Saisto et. al [27] обнаружилось, что на психологическое благополучие женщины после рождения ребенка оказывают влияние такие параметры, как чувство безопасности и наличие поддержки во время родов, возможность самостоятельно принимать решения. K. I. Mohammad et. al [28] говорят также о важности уважения к женщине и ее личному пространству, отсутствии стимуляции и ускорения процесса родов без медицинской необходимости. Осложнения в родах часто становятся для женщины неожиданностью, стрессом, который может провоцировать тяжелые переживания и чувство вины.

В современном российском обществе количество нуклеарных семей возрастает, повышаются требования к качеству супружеских отношений [29]. В связи с этим особое значение и ценность для матери имеет поддержка супруга, а не других родственников. Образ жизни женщины меняется, забота о ребенке требует серьезных эмоциональных и физических ресурсов. В этой ситуации женщине важно не оставаться наедине с трудностями заботы о ребенке, а иметь поддержку супруга [30].

В нашей работе выделены только несколько психологических и физиологических условий, способствующих развитию послеродовой депрессии. Нам представляется продуктивным дальнейшее исследование факторов риска развития депрессии, связанных с отношением женщины к ребенку и к себе в роли матери, с качеством супружеских отношений, ожиданиями относительно материнства и роли супруга и т. д. Выделение группы риска имеет значение для профилактики развития депрессии после рождения ребенка. Одним из наиболее эффективных способов совладания с депрессией является получение родителями профессиональной поддержки и информирование о симптомах заболевания на этапе беременности [4]. Проблема диагностики, профилактики и послеродового патронажного сопровождения, несомненно, является социально значимой.

\section{Литература}

1. Ramadas S., Kumar R. Postnatal depression: a narrative review // International Journal of Culture and Mental Health. - 2016. - Vol. 9, № 2. - P. 97-107. DOI: 10.1080/17542863.2015.1065893

2. Fuggle P., Glover L., Khan F., Haydon K. Screening for postnatal depression in Bengali women: Preliminary observations from using a translated version of 
the Edinburgh Postnatal Depression Scale (EPDS) // Journal of Reproductive and Infant Psychology. - 2002. - Vol. 20, № 2. - P. 71-82.

3. Green K., Broome H., Mirabella J. Postnatal depression among mothers in the United Arab Emirates: Socio-cultural and physical factors // Psychology, Health \& Medicine. - 2006. - Vol. 11, № 4. - P.425-431.DOI: 10.1080/13548500600678164

4. Boath E., Henshaw C. The treatment of postnatal depression: A comprehensive literature review // Journal of Reproductive and Infant Psychology. - 2001. Vol. 19, № 3. - P. 215-248. DOI: 10.1080/02646830120073224

5. McMahon C., Boivin J., Gibson F. L., Hammarberg K., Wynter L., Fisher J. Older maternal age and major depressive episodes in the first two years after birth: Findings from the Parental Age and Transition to Parenthood Australia (PATPA) study // Journal of Affective Disorders. - 2015. - Vol. 175. P. 454-462. DOI: 10.1016/j.jad.2015.01.025

6. Bell A. F., Andersson E. The birth experience and women's postnatal depression: A systematic review // Midwifery. - 2016. - Vol. 39. - P. 112-123. DOI: 10.1016/j.midw.2016.04.014

7. O'Hara M. W., Wisner K. L. Perinatal mental illness: definition, description and etiology // Best Practice \& Research: Clinical Obstetrics \& Gynaecology. 2014. - Vol. 28, Issue 1. - P. 3-12. DOI: 10.1016/j.bpobgyn.2013.09.002

8. Thompson K. D., Bendell D. Depressive cognitions, maternal attitudes and postnatal depression // Journal of Reproductive and Infant Psychology. 2014. - Vol. 32, Issue 1. - P. 70-82. DOI: 10.1080/02646838.2013.858312

9. FranckE., Vanderhasselt M.-A., Goubert L., Loeys T., Temmerman M., De Raedt R. The role of self-esteem instability in the development of postnatal depression: A prospective study testing a diathesis-stress account // Journal of Behavior Therapy \& Experimental Psychiatry. - 2016. - Vol. 50. - P. 15-22. DOI: 10.1016/j.jbtep.2015.04.010

10. DeJong H., Fox E., Stein A. Rumination and postnatal depression: A systematic review and cognitive model // Behaviour Research and Therapy. - 2016. Vol. 82. - P. 38-49. DOI: 10.1016/j.brat.2016.05.003

11. Rutherford H. J. V., Wallace N. S., Laurent H. K., Mayes L. C. Emotion regulation in parenthood // Developmental Review. - 2015. - Vol. 36. - P. 1-14. DOI: 10.1016/j.dr.2014.12.008

12. Deater-Deckard K., Wang Z., Chen N., Bell M. A. Maternal executive function, harsh parenting, and child conduct problems // The Journal of Child Psychology and Psychiatry. - 2012. - Vol. 53, № 10. - P. 1084-1091. DOI: 10.1111/j.1469-7610.2012.02582.x

13. Wilkinson R. B., Mulcahy R. Attachment and interpersonal relationships in postnatal depression // Journal of Reproductive and Infant Psychology. 2010. - Vol. 28, Issue 3. - P. 252-265. DOI: 10.1080/02646831003587353 
14. Якупова В. А. Психологические условия успешного освоения материнской роли // Консультативная психология и психотерапия. - 2017. - Т. 25, № 1. - C. 59-71. DOI: 10.17759/cpp.2017250105

15. Murray L., Cooper P. EDITORIAL: Postpartum depression and child development // Psychological Medicine. - 1997. - Vol. 27, Issue 2. - P. 253-260.

16. Murray L., Sinclair D., Cooper P., Ducournau P., Turner P., Stein A. The Socioemotional Development of 5-year-old Children of Postnatally Depressed Mothers // Journal of Child Psychology and Psychiatry. - 1999. - Vol. 40, Issue 8. - P. 1259-1271. DOI: 10.1111/1469-7610.00542

17. Murray L. The Impact of Postnatal Depression on Infant Development // Journal of Child Psychology and Psychiatry. - 1992. - Vol. 33, Issue 3. P. 543-561. DOI: 10.1111/j.1469-7610.1992.tb00890.x

18. Nolvi S., Karlsson L., Bridgett D. J., Pajulo M., Tolvanen M., Karlsson H. Maternal postnatal psychiatric symptoms and infant temperament affect early mother-infant bonding // Infant Behavior and Development. - 2016. Vol. 43. - P. 13-23. DOI: 10.1016/j.infbeh.2016.03.003

19. Beestin L., Hugh-Jones S., Gough B. The impact of maternal postnatal depression on men and their ways of fathering: an interpretative phenomenological analysis // Psychology \& Health. - 2014. - Vol. 29, Issue 6. - P. 717-735. DOI: 10.1080/08870446.2014.885523

20. Bielawska-Batorowicz E., Kossakowska-Petrycka K. Depressive mood in men after the birth of their offspring in relation to a partner's depression, social support, fathers' personality and prenatal expectations // Journal of Reproductive and Infant Psychology. - 2006. - Vol. 24, Issue 1. - P. 21-29. DOI: $10.1080 / 02646830500475179$

21. Beck A. T., Steer R. A., Garbin M. G. Psychometric properties of the Beck Depression Inventory: Twenty-five years of evaluation // Clinical Psychology Review. - 1988. - Vol. 8, Issue 1. - P. 77-100. DOI: 10.1016/0272-7358(88)90050-5

22. CoxJ.L., Holden J.M., Sagovsky R. Detection of Postnatal Depression: Development of the 10-item Edinburgh Postnatal Depression Scale // The British Journal of Psychiatry. - 1987. - Vol. 150, Issue 6. - P. 782-786. DOI: 10.1192/bjp.150.6.782

23. Ильин Е. П. Эмоции и чувства. - СПб. : Питер, 2001. - 752 с.

24. Каппони В., Новак Т. Сам себе психолог. - СПб. : Питер, 1994. - 122 с.

25. Мазо Г. Э., Вассерман Л. И., Шаманина М. В. Выбор шкал для оценки послеродовой депрессии // Обозрение психиатрии и медицинской психологии имени В. М. Бехтерева. - 2012. - № 2. - С. 41-50.

26. Muscat T., Thorpe K., Obst P. Disconfirmed expectations of infant behaviours and postnatal depressive symptoms among parents // Journal of Reproductive and Infant Psychology. - 2012. - Vol. 30, Issue 1. - P. 51-61. - DOI: 10.1080/02646838.2012.670804 


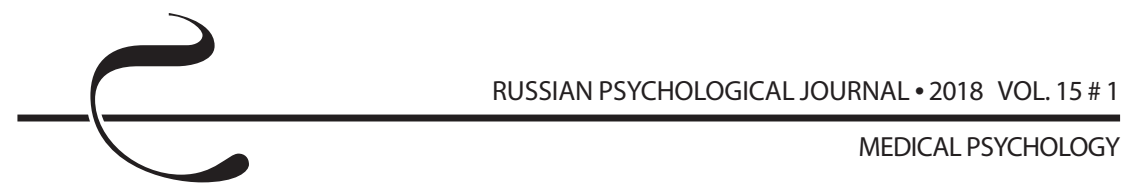

27. Saisto T., Salmela-Aro K., Nurmi J.-E., Halmesmäki E. Psychosocial predictors of disappointment with delivery and puerperal depression. A longitudinal study // Acta Obstetricia et Gynecologica Scandinavica. - 2001. - Vol. 80, Issue 1. - P. 39-45. DOI: 10.1034/j.1600-0412.2001.800108.x

28. Mohammad K. I., Gamble J., Creedy D. K. Prevalence and factors associated with the development of antenatal and postnatal depression among Jordanian women // Midwifery. - 2011. - Vol. 27, Issue 6. - P. e238-e245. DOI: 10.1016/j.midw.2010.10.008

29. Гурко Т. А. Трансформация брачно-семейных отношений // Россия: трансформирующееся общество / под ред. В. А. Ядова. - М. : КАНОНпресс-Ц, 2001. - 640 с.

30. Schoppe-Sullivan S. J., Settle T., Lee J.-K., Kamp Dush C. M. Supportive Coparenting Relationships as a Haven of Psychological Safety at the Transition to Parenthood // Research in Human Development. - 2016. - Vol. 13, Issue 1. - P. 32-48. DOI: 10.1080/15427609.2016.1141281

\section{References}

1. Ramadas S., Kumar R. Postnatal depression: a narrative review. International Journal of Culture and Mental Health, 2016, V. 9, no. 2, pp. 97-107. DOI: 10.1080/17542863.2015.1065893

2. Fuggle P., Glover L., Khan F., Haydon K. Screening for postnatal depression in Bengali women: Preliminary observations from using a translated version of the Edinburgh Postnatal Depression Scale (EPDS). Journal of Reproductive and Infant Psychology, 2002, V. 20, no. 2, pp. 71-82.

3. Green K., Broome H., Mirabella J. Postnatal depression among mothers in the United Arab Emirates: Socio-cultural and physical factors. Psychology, Health \& Medicine, 2006, V. 11, no. 4, pp. 425-431. DOI: 10.1080/13548500600678164

4. Boath $E$., Henshaw C. The treatment of postnatal depression: A comprehensive literature review. Journal of Reproductive and Infant Psychology, 2001, V. 19, no. 3, pp. 215-248. DOI: 10.1080/02646830120073224

5. McMahon C., Boivin J., Gibson F. L., Hammarberg K., Wynter L., Fisher J. Older maternal age and major depressive episodes in the first two years after birth: Findings from the Parental Age and Transition to Parenthood Australia (PATPA) study. Journal of Affective Disorders, 2015, V. 175, pp. 454462. DOI: 10.1016/j.jad.2015.01.025

6. Bell A. F., Andersson E. The birth experience and women's postnatal depression: A systematic review. Midwifery, 2016, V. 39, pp. 112-123. DOI: 10.1016/j.midw.2016.04.014

7. O'Hara M. W., Wisner K. L. Perinatal mental illness: definition, description and etiology. Best Practice \& Research:Clinical Obstetrics \& Gynaecology, 2014, 


\section{28, Issue 1, pp. 3-12. DOI: 10.1016/j.bpobgyn.2013.09.002}

8. Thompson K. D., Bendell D. Depressive cognitions, maternal attitudes and postnatal depression. Journal of Reproductive and Infant Psychology, 2014, V. 32, Issue 1, pp. 70-82. DOI: 10.1080/02646838.2013.858312

9. Franck E., Vanderhasselt M.-A., Goubert L., Loeys T., Temmerman M., De Raedt R. The role of self-esteem instability in the development of postnatal depression: A prospective study testing a diathesis-stress account. Journal of BehaviorTherapy \& Experimental Psychiatry, 2016, V. 50, pp. 15-22. DOI: 10.1016/j.jbtep.2015.04.010

10. DeJong H., Fox E., Stein A. Rumination and postnatal depression: A systematic review and cognitive model. Behaviour Research and Therapy, 2016, V. 82, pp. 38-49. DOI: 10.1016/j.brat.2016.05.003

11. Rutherford H. J. V., Wallace N. S., Laurent H. K., Mayes L. C. Emotion regulation in parenthood. Developmental Review, 2015, V. 36, pp. 1-14. DOI: 10.1016/j.dr.2014.12.008

12. Deater-Deckard K., Wang Z., Chen N., Bell M. A. Maternal executive function, harsh parenting, and child conduct problems. The Journal of Child Psychology and Psychiatry, 2012, V. 53, no. 10, pp. 1084-1091. DOI: 10.1111/j.1469-7610.2012.02582.x

13. Wilkinson R. B., Mulcahy R. Attachment and interpersonal relationships in postnatal depression. Journal of Reproductive and Infant Psychology, 2010, V. 28, Issue 3, pp. 252-265. DOI: 10.1080/02646831003587353

14. Yakupova V. A. Psychological conditions for maternal role attainment. Konsul'tativnaya psikhologiya i psikhoterapiya - Counseling Psychology and Psychotherapy, 2017, V. 25, no. 1, pp. 59-71 (in Russian). DOI: 10.17759/ cpp.2017250105

15. Murray L., Cooper P. EDITORIAL: Postpartum depression and child development. Psychological Medicine, 1997, V. 27, Issue 2, pp. 253-260.

16. Murray L., Sinclair D., Cooper P., Ducournau P., Turner P., Stein A. The socioemotional development of 5-year-old children of postnatally depressed mothers. Journal of Child Psychology and Psychiatry, 1999, V. 40, Issue 8, pp. 1259-1271. DOI: 10.1111/1469-7610.00542

17. Murray L. The impact of postnatal depression on infant development. Journal of Child Psychology and Psychiatry, 1992, V. 33, Issue 3, pp. 543-561. DOI: 10.1111/j.1469-7610.1992.tb00890.x

18. Nolvi S., Karlsson L., Bridgett D. J., Pajulo M., Tolvanen M., Karlsson H. Maternal postnatal psychiatric symptoms and infant temperament affect early mother-infant bonding. Infant Behavior and Development, 2016, V. 43, pp. 13-23. DOI: 10.1016/j.infbeh.2016.03.003

19. Beestin L., Hugh-Jones S., Gough B. The impact of maternal postnatal depression on men and their ways of fathering: an interpretative 
phenomenological analysis. Psychology \& Health, 2014, V. 29, Issue 6, pp. 717-735. DOI: 10.1080/08870446.2014.885523

20. Bielawska-Batorowicz E., Kossakowska-Petrycka K. Depressive mood in men after the birth of their offspring in relation to a partner's depression, social support, fathers' personality and prenatal expectations. Journal of Reproductive and Infant Psychology, 2006, V. 24, Issue 1, pp. 21-29. DOI: 10.1080/02646830500475179

21. Beck A. T., Steer R. A., Garbin M. G. Psychometric properties of the Beck Depression Inventory: Twenty-five years of evaluation. Clinical Psychology Review, 1988, V. 8, Issue 1, pp. 77-100. DOI: 10.1016/0272-7358(88)90050-5

22. Cox J. L., Holden J. M., Sagovsky R. Detection of postnatal depression: Development of the 10-item Edinburgh Postnatal Depression Scale. The British Journal of Psychiatry, 1987, V. 150, Issue 6, pp. 782-786. DOI: 10.1192/ bjp.150.6.782

23. Il'in E. P. Emotsii i chuvstva [Emotions and feelings]. St. Petersburg, Piter Publ., 2001. $752 \mathrm{p}$.

24. Kapponi V., Novak T. Sam sebe psikholog [A psychologist by nature]. St. Petersburg, Piter Publ., 1994. 122 p.

25. Mazo G. E., Vasserman L. I., Shamanina M. V. Choosing scales for assessing postnatal depression. Obozrenie psikhiatrii i meditsinskoi psikhologii imeni V. M. Bekhtereva - The Bekhterev Review of Psychiatry and Medical Psychology, 2012, no. 2, pp. 41-50 (in Russian).

26. Muscat T., Thorpe K., Obst P. Disconfirmed expectations of infant behaviours and postnatal depressive symptoms among parents. Journal of Reproductive and Infant Psychology, 2012, V. 30, Issue 1, pp. 51-61. DOI: 10.1080/02646 838.2012.670804

27. Saisto T., Salmela-Aro K., Nurmi J.-E., Halmesmäki E. Psychosocial predictors of disappointment with delivery and puerperal depression. A longitudinal study. Acta Obstetricia et Gynecologica Scandinavica, 2001, V. 80, Issue 1, pp. 39-45. DOI: 10.1034/j.1600-0412.2001.800108.x

28. Mohammad K. I., Gamble J., Creedy D. K. Prevalence and factors associated with the development of antenatal and postnatal depression among Jordanian women. Midwifery, 2011, V. 27, Issue 6, pp. e238-e245. DOI: 10.1016/j.midw.2010.10.008

29. Gurko T. A. Transformation of family and marriage relationships. In: Yadov V. A. (ed.) Rossiya: transformiruyushcheesya obshchestvo [Russia: a transforming society]. Moscow, KANON-press-Ts Publ., 2001. 640 p.

30. Schoppe-Sullivan S. J., Settle T., Lee J.-K., Kamp Dush C. M. Supportive coparenting relationships as a haven of psychological safety at the transition to parenthood. Research in Human Development, 2016, V. 13, Issue 1, pp. 32-48. DOI: 10.1080/15427609.2016.1141281 